\title{
Extremal principle of entropy production in the climate system
}

\author{
By T. PUJOL ${ }^{1 *}$ and J. E. LLEBOT ${ }^{2}$ \\ ${ }^{1}$ Universitat de Girona, Spain \\ ${ }^{2}$ Universitat Autònoma de Barcelona, Spain
}

(Received 12 August 1997; revised 16 June 1998)

\begin{abstract}
SUMMARY
Paltridge found reasonable values for the most significant climatic variables through maximizing the material transport part of entropy production by using a simple box model. Here, we analyse Paltridge's box model to obtain the energy and the entropy balance equations separately. Derived expressions for global entropy production, which is a function of the radiation field, and even its material transport component, are shown to be different from those used by Paltridge. Plausible climatic states are found at extrema of these parameters. Feasible results are also obtained by minimizing the radiation part of entropy production, in agreement with one of Planck's results. Finally, globally averaged values of the entropy flux of radiation and material entropy production are obtained for two dynamical extreme cases: an earth with uniform temperature, and an earth in radiative equilibrium at each latitudinal point.
\end{abstract}

KeYwords: Box-model Climate Entropy Extremal dissipation principle

\section{INTRODUCTION}

Simple climate models have been used in order to understand the role of entropy production in the climate. In such an open system Prigonine's theorem of minimum dissipation at the earth's climatic steady state is not, in principle, expected to be valid (Prigogine 1947; De Groot and Mazur 1984). However, the existence of an extremal condition for the entropy production would be desirable, because it would generate an additional constraint for the variables that describe the system. In low-dimensional models, where the number of free variables is very limited, an extremal condition would restrict the possible states reached by the system and would depend on the values obtained for the unknowns. Paltridge $(1975,1978,1979,1981)$ developed a zonally averaged climate box model, where temperature, cloud cover, meridional heat flux and latent- plus sensible-heat flux were obtained through maximising the material transport part of entropy production. The results found for these parameters showed surprisingly good agreement with the observations. In the light of these encouraging results, there was a substantial increase in studies involving entropy production as a fundamental component of climate behaviour (e.g. Grassl 1981; Shutts 1981; Mobbs 1982; Wyant et al. 1988), although there was no justification for the maximum-dissipation hypothesis.

However, a thermodynamic description of the global entropy production for the climate should not only take the material part into account but also the radiative contribution. Historically, the thermodynamic study of radiative processes has been a controversial area of study, due to the discussion on whether radiative entropy production has a bilinear form and, in consequence, whether extremal principles obtained by classical irreversible thermodynamics can be applied. In fact, when the rate of entropy production includes the radiation field, it clearly seems to be non-bilinear. Therefore, some authors (e.g. Callies and Herbert 1984, 1988) have proposed a bilinear form for the radiative contribution by using effective temperatures linked to the entropy flux of radiation. The problems arise from the fact that the entropy flux of radiation is not linearly related to the energy flux of radiation (Ore 1955; Landsberg and Tonge 1979); and also from the fact that the existence of a phenomenological law linking the radiative flux with a given thermodynamic force is not clearly established. However, one can find some studies (e.g. Ciancio and Verhás

* Corresponding author: Departament de Fisica, Universitat de Girona, Campus Montilivi 17071 Girona, Catalonia, Spain. 


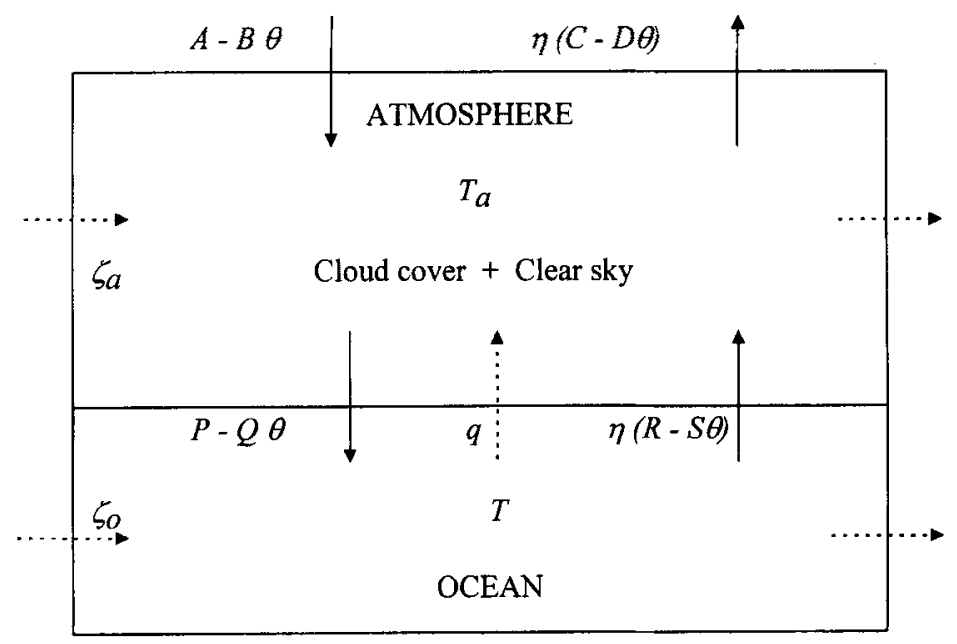

Figure 1. Schematic view of one cell of the ten-box Paltridge's model. Free variables are: temperature $T$, cloud cover $\theta$, latent- and sensible-heat fluxes $\mathscr{L} \mathscr{E}+\mathscr{H}$, and meridional heat flux $X . T_{\mathrm{a}}$ is the atmospheric temperature. Following O'Brien and Stephens (1995): $\eta=\sigma T^{4} / F, \zeta=\Delta X / F=\zeta_{\mathrm{a}}+\zeta_{\mathrm{o}}$ (divergence of meridional heat flux) and $q=(\mathscr{L} \mathscr{E}+\mathscr{H}) / F$ where $F$ is the solar constant and $\sigma$ the Stefan's constant. The parameters $A, B, P$ and $Q$ related to the short-wave radiation are defined as: $A=\varepsilon\left(1-\omega_{\mathrm{g}}\right), B=\varepsilon\left(\omega_{\mathrm{c}}-\omega_{\mathrm{g}}\right), P=\varepsilon\left(1-\omega_{\mathrm{g}}-\alpha_{\mathrm{a}}\right)$ and $Q=\varepsilon\left(\omega_{\mathrm{c}}+\alpha_{\mathrm{c}}-\omega_{\mathrm{g}}\right)$, and are all positive with $\omega_{\mathrm{g}}, \omega_{\mathrm{c}}, \alpha_{\mathrm{a}}$ and $\alpha_{\mathrm{c}}$ the clear-sky albedo, cloud albedo, atmospheric absorption and cloud absorption respectively; $\varepsilon$ is the ratio between the incoming and emitting surfaces. The parameters of long-wave radiation are: $C=\left(m_{\mathrm{g}}+m_{\mathrm{a}}\right), D=\left(m_{\mathrm{g}}+m_{\mathrm{a}}-m_{\mathrm{c}}\right), R=m_{\mathrm{g}}$ and $S=n_{\mathrm{c}}$, and are all positive with $m_{\mathrm{g}}, m_{\mathrm{a}}, m_{\mathrm{c}}$ and $n_{\mathrm{c}}$ the surface, clear-sky, cloud and cloud base emissivities.

1990,1991 ) where a constitutive law for atmospheric radiation is used, which is based on the radiative-transfer equation.

Even so, taking the radiation in the atmospheric system into account, Planck (1913) obtained a state of minimum radiative entropy production at stationary conditions, assuming a plane-parallel isothermal atmosphere with an isotropic radiation field. Based on this work, Essex (1984a) also obtained a minimum rate for radiative entropy production by assuming a grey atmosphere in radiative equilibrium. With reference to thermodynamic climate models, Essex (1984b) considers global entropy production to be the entropy flux of radiation, which is found to be 100 times more important than the material entropy contribution alone and at least eight times more sensitive to the fundamental mode of climate perturbations. This fact is also observed in a one dimensional (1D) horizontal model by $\mathrm{Li}$ and Chýlek (1994) and in a 1D vertical model by Li et al. (1994). Some other authors (e.g. Nicolis and Nicolis 1980) only consider material entropy production when studying the behaviour of the atmospheric entropy, arguing that only a small contribution of the entropy flux of radiation is associated with dynamical processes. In reference to Paltridge's box model (see Fig. 1), the maximum-dissipation hypothesis has only been applied to the material part of entropy production $\sigma_{\mathrm{m}}$, which is accepted as $\zeta / T_{\mathrm{a}}$, where $T_{\mathrm{a}}$ is the atmospheric temperature according to Paltridge's procedure, and $\zeta$ the meridional divergence of heat flux (Paltridge 1975, 1978; Grassl 1981; O'Brien and Stephens 1995). This definition, for example, produces the conceptual problem of no $\sigma_{\mathrm{m}}$ in a $0 \mathrm{D}$ model.

Therefore, in the present paper, $\sigma_{\mathrm{m}}$ has been modified by taking all the possible dissipation processes in this type of model into account. Thus, material entropy production includes, individually, the contributions of meridional atmospheric and oceanic heat fluxes as well as that provided by latent-plus sensible-heat flux. Then, a non-zero value is obtained in a globally averaged model. Hence, $\sigma_{\mathrm{m}}$ can be studied in the same way as the entropy flux 
of radiation. Furthermore, we show the possible application of the maximum-dissipation hypothesis to global entropy production $\sigma_{\mathrm{g}}$.

\section{EXTREMA OF ENTROPY PRODUCTION}

The Paltridge's box model used in the present study contains ten latitudinal cells of equal surface area, each one divided into two sections: an oceanic and an atmospheric part; a schematic view of one cell is given in Fig. 1. The model follows the accurate analysis carried out by O'Brien and Stephens (1995). Two energy balance equations are obtained for each cell. The further two equations needed to complete the system to resolve the four unknowns are provided by the convective hypothesis and the principle of maximum entropy production. O'Brien and Stephens (1995) show that the convective hypothesis implies a simple analytical relationship between temperature, cloud-cover and meridional heat fluxes. This hypothesis is based on the idea that the atmospheric system will evolve to a state of maximum latent- plus sensible-heat flux (maximum possible convective processes, an assumption strongly related to dynamical considerations). Once, by means of the convective hypothesis, the temperature $T$ and cloud cover $\theta$ are linked with $\zeta$, the climate state is found at extrema of the global, material or radiative $\sigma_{\mathrm{r}}$ entropy production (where $\sigma_{\mathrm{r}}=\sigma_{\mathrm{g}}-\sigma_{\mathrm{m}}$ ). O'Brien and Stephens (1995) also showed that the extremal process proposed by Paltridge can be reduced to finding an extremum of a set of ten independent equations (one for each cell), only constrained by the condition of zero storage of heat flux over the whole system. Details of this calculation are given in the appendix.

For an individual box (see Fig. 1), the energy balance equation at stationary conditions leads to:

$$
-\nabla \cdot \mathbf{H}-\nabla \cdot \mathbf{Q}=0
$$

where $\mathbf{H}$ and $\mathbf{Q}$ are the radiative and material energy fluxes respectively. Equation (1) applied to the atmosphere and to the ocean, normalized by solar constant and surface area, gives:

$$
\begin{gathered}
(A-B \theta)-(P-Q \theta)-\eta(C-D \theta)+\eta(R-S \theta)+\zeta_{\mathrm{a}}+q=0, \\
P-Q \theta-\eta(R-S \theta)+\zeta_{\mathrm{o}}-q=0 .
\end{gathered}
$$

Free variables are $T, \theta$, latent- and sensible-heat fluxes $\mathscr{L} \mathscr{E}+\mathscr{H}$, and meridional heat flux $X$. Following O'Brien and Stephens (1995): $\eta=\sigma T^{4} / F, \zeta=\Delta X / F=\zeta_{\mathrm{a}}+\zeta_{\mathrm{o}}$ and $q=(\mathscr{L} \mathscr{E}+\mathscr{H}) / F$, where $F$ is the solar constant and $\sigma$ the Stefan's constant. The parameters $A, B, P$ and $Q$ are related to the short-wave radiation and defined as: $A=$ $\varepsilon\left(1-\omega_{\mathrm{g}}\right), B=\varepsilon\left(\omega_{\mathrm{c}}-\omega_{\mathrm{g}}\right), P=\varepsilon\left(1-\omega_{\mathrm{g}}-\alpha_{\mathrm{a}}\right)$ and $Q=\varepsilon\left(\omega_{\mathrm{c}}+\alpha_{\mathrm{c}}-\omega_{\mathrm{g}}\right)$; they are all positive with $\omega_{\mathrm{g}}, \omega_{\mathrm{c}}, \alpha_{\mathrm{a}}$ and $\alpha_{\mathrm{c}}$ the clear-sky albedo, cloud albedo, atmospheric absorption and cloud absorption respectively; $\varepsilon$ is the ratio between the incoming and emitting surfaces. The parameters of long-wave radiation are: $C=\left(m_{\mathrm{g}}+m_{\mathrm{a}}\right), D=\left(m_{\mathrm{g}}+m_{\mathrm{a}}-m_{\mathrm{c}}\right)$, $R=m_{\mathrm{g}}$ and $S=n_{\mathrm{c}}$; they are all positive with $m_{\mathrm{g}}, m_{\mathrm{a}}, m_{\mathrm{c}}$ and $n_{\mathrm{c}}$ the surface, clear-sky, cloud and cloud base emissivities. $\sigma_{\mathrm{g}}$ is obtained from the entropy balance equation and the second law of thermodynamics, from which the general expression is given by (Lesins 1990; Peixoto et al. 1991; Li and Chýlek 1994):

$$
\sigma_{\mathrm{g}}=\nabla \cdot \mathbf{J}-\frac{\nabla \cdot \mathbf{H}}{T}-\frac{\nabla \cdot \mathbf{Q}}{T},
$$

where $\mathbf{J}$ is the entropy flux of radiation. The material entropy flux $\nabla \cdot(\mathbf{Q} / T)$ has been removed in (3) because it vanishes on global integration using the boundary conditions: $\mathbf{Q}($ North Pole $)=\mathbf{Q}($ South Pole $)=0$. 
Then, by assuming the atmospheric temperature $T_{\mathrm{a}}$ and surface temperature $T$ as those characteristic for the atmosphere and ocean part respectively, the equations for the global entropy production of the atmosphere $\sigma_{\mathrm{ga}}$, and ocean $\sigma_{\mathrm{go}}$, normalised by solar constant and surface area, yield:

$$
\begin{gathered}
\sigma_{\mathrm{ga}}=\nabla \cdot \mathbf{J}_{\mathrm{a}}-\frac{\eta(C-D \theta)-\eta(R-S \theta)}{T_{\mathrm{a}}}+\frac{(A-B \theta)-(P-Q \theta)}{T_{\mathrm{a}}}+\frac{\zeta_{\mathrm{a}}}{T_{\mathrm{a}}}+\frac{q}{T_{\mathrm{a}}}, \\
\sigma_{\mathrm{go}}=\nabla \cdot \mathbf{J}_{\mathrm{o}}-\frac{\eta(R-S \theta)}{T}+\frac{P-Q \theta}{T}+\frac{\zeta_{\mathrm{o}}}{T}-\frac{q}{T} .
\end{gathered}
$$

Since the specific entropy intensity is not linearly related to the specific energy intensity (Landsberg and Tonge 1979; Stephens and O'Brien 1993; Goddy and Abdou 1996; O'Brien 1997), the entropy flux density $J$ of a surface with emissivity $\omega$ follows:

$$
J=\frac{4}{3} \sigma T^{3} \chi(\omega),
$$

where $0 \leqslant \chi(\omega) \leqslant 1$ with $\chi(1)=1$ and $\chi(0)=0$.

Stephens and O'Brien (1993) show that the substitution of $\chi(\omega)$ for $\omega$ is acceptable as an approximation for high values of $\omega$ (e.g. in emitting surfaces). In this case, an expression linking the entropy with the energy flux of radiation through an effective temperature, $T_{\text {eff }}$, of the system ( $T$ of each box for the emitting radiation) can be assumed (Li and Chýlek 1994):

$$
\nabla \cdot \mathbf{J}=\frac{4}{3} \frac{\nabla \cdot \mathbf{H}}{T_{\mathrm{eff}}} .
$$

However, for very low values of $\omega$, as is the case for reflected incoming short-wave radiation, $\chi(\omega)$ differs substantially from $\omega$ and (6) becomes incorrect. Stephens and O'Brien (1993) obtain the following parametrization for $\chi(\omega)$ to be applied to reflected short-wave entropy radiation:

$$
\chi(\omega)=\omega\{u \log (\omega)+\nu\},
$$

with $u \approx-0.277$ and $v \approx 0.965$.

In using (7), Stephens and O'Brien (1993) note that the globally averaged short-wave entropy flux of radiation is positive (outgoing) in observations by satellite. This is not possible if (6) is accepted for the reflected short-wave entropy.

Hence, the values of $\nabla \cdot \mathbf{J}_{\mathrm{ga}}$ and $\nabla \cdot \mathbf{J}_{\mathrm{go}}$ normalised by solar constant and surface area, become:

$$
\begin{aligned}
\nabla \cdot \mathbf{J}_{\mathrm{ga}}= & \frac{4}{3} \frac{\eta(C-D \theta)-\eta(R-S \theta)}{T_{\mathrm{a}}}-\frac{4}{3} \frac{\varepsilon}{T_{\text {sun }}}\left(1-\theta \psi\left(\omega^{\mathrm{c}}\right)-(1-\theta) \psi\left(r \omega^{\mathrm{g}}\right)\right) \\
+ & \frac{4}{3} \frac{(P-Q \theta)}{T_{\text {sun }}}, \\
& \nabla \cdot \mathbf{J}_{\mathrm{go}}=\frac{4}{3} \frac{\eta(R-S \theta)}{T}-\frac{4}{3} \frac{(P-Q \theta)}{T_{\text {sun }}},
\end{aligned}
$$

where the first term including the $4 / 3$ factor corresponds to the long-wave entropy radiation, and the second term refers to the short-wave contribution at the top of the atmosphere which has been split into the entropy radiation reflected by clouds $\psi\left(\omega^{\mathrm{c}}\right)$ and that reflected by clear sky $\psi\left(\omega^{\mathfrak{g}}\right) . \omega^{\mathfrak{c}, g}=\omega_{\mathrm{c}, \mathrm{g}} \Omega \cos \xi$, with $\xi$ the solar zenith angle, $\Omega$ is the solid angle 


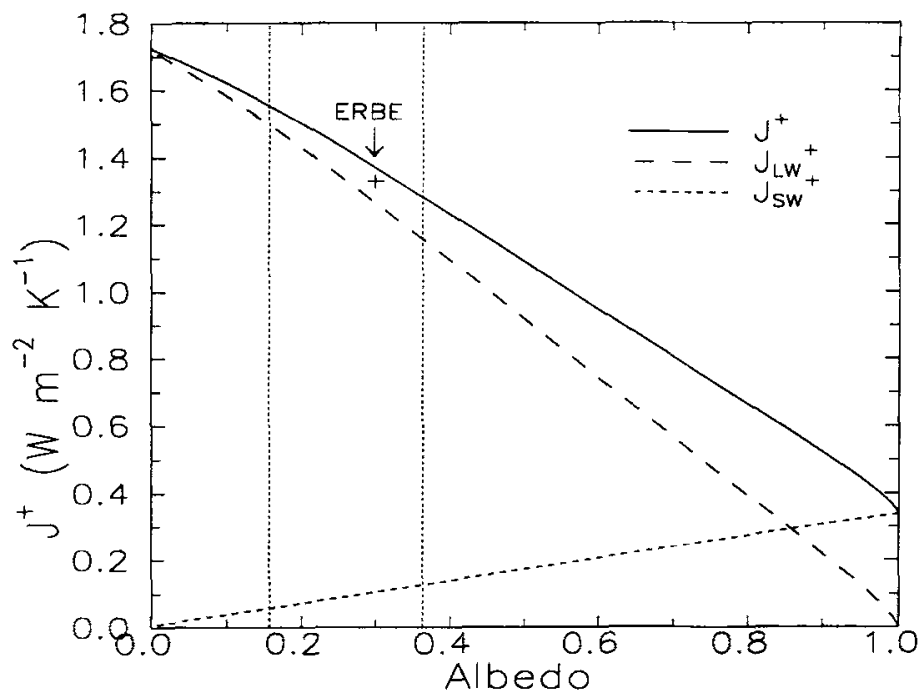

Figure 2. Globally averaged values of total outgoing entropy flux density, $J^{+}$, and its long-wave, $J_{\mathrm{LW}}^{+}$, and shortwave, $J_{\mathrm{SW}}^{+}$, contribution. Dotted vertical lines indicate the expected range for the values within the Paltridge's box-model $(0 \leqslant$ cloud-cover $\leqslant 1)$. The measure of $J^{+}$by satellite, ERBE (see text), is also shown (Stephens and O’Brien 1993).

subtended by the sun, and $\omega_{c, g}$ the cloudy and clear sky albedo at the top of the atmosphere, respectively. $T_{\text {sun }}$ has been assumed $\approx 5777 \mathrm{~K}$. Moreover, in (8a) and (8b):

$$
\psi\left(\omega^{\mathrm{c}, \mathrm{g}}\right)=\omega_{\mathrm{c}, \mathrm{g}}\left\{u \log \left(2 \omega_{\mathrm{c}, \mathrm{g}} \Omega \varepsilon \pi\right)+\nu-u\right\},
$$

because $\chi(\omega)$ has been integrated along the longitude, keeping its latitudinal dependence (see for instance O'Brien 1997). At the surface there is no term involving the factor $\chi$ because short-wave radiation has been assumed to be entirely incoming.

At the steady state, entropy production is only related to the radiative entropy flux, the only component which crosses the boundary of the system. Then, from (4a), (4b) and (8a), (8b) global entropy production $\sigma_{\mathrm{g}}=\sigma_{\mathrm{ga}}+\sigma_{\mathrm{go}}$ may be written in the form:

$$
\begin{gathered}
\sigma_{\mathrm{g}}=\frac{4}{3} \frac{\eta(C-D \theta)}{T_{\mathrm{a}}}-\frac{4}{3} \frac{\varepsilon}{T_{\text {sun }}}\left\{1-\theta \psi\left(\omega^{\mathrm{c}}\right)-(1-\theta) \psi\left(\omega^{\mathrm{g}}\right)\right\} \\
-\frac{4}{3} \eta(R-S \theta)\left(\frac{1}{T_{\mathrm{a}}}-\frac{1}{T}\right),
\end{gathered}
$$

which clearly differs from that related to the material part, which from (4a) and (4b) takes the form:

$$
\sigma_{\mathrm{m}}=\frac{\zeta_{\mathrm{a}}}{T_{\mathrm{a}}}+\frac{\zeta_{\mathrm{o}}}{T}+q\left(\frac{1}{T_{\mathrm{a}}}-\frac{1}{T}\right) .
$$

This expression is not exactly the same as that used by Paltridge in his box model (i.e. $\zeta / T_{\mathrm{a}}$; Paltridge 1978).

Globally averaged values can be obtained from the above expressions without applying the maximum-dissipation hypothesis. In Fig. 2, the entropy flux density of radiation at the top of the atmosphere is shown as a function of the planetary albedo. $J^{+}$represents the total outgoing entropy flux density. $J_{\mathrm{SW}}^{+}$and $J_{\mathrm{LW}}^{+}$are the outgoing contribution of the 


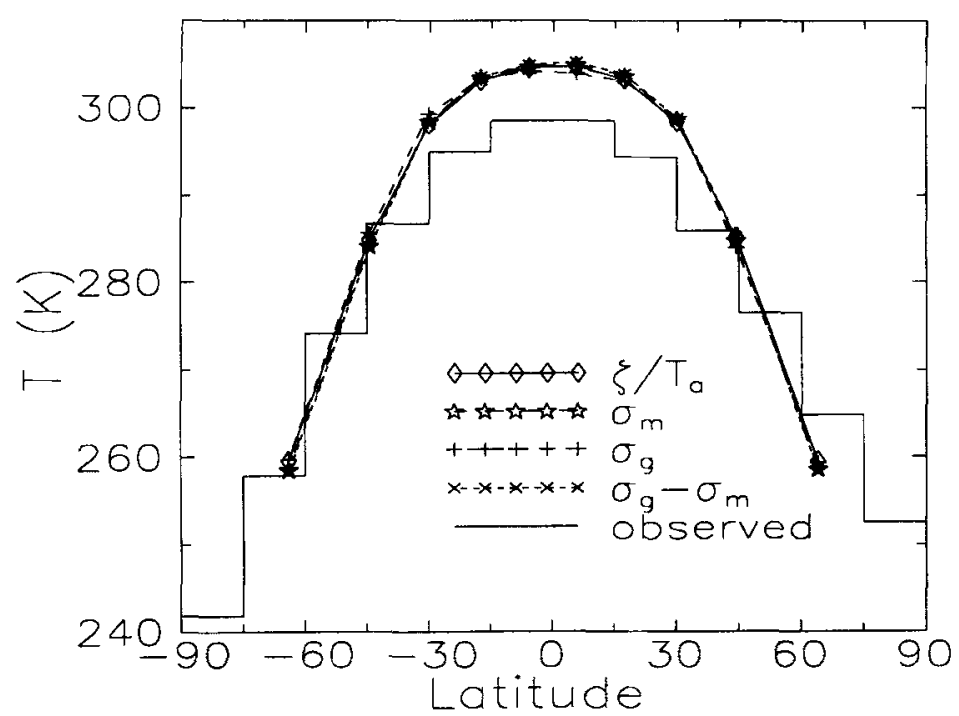

Figure 3. Temperature $T$ obtained at extrema of global $\sigma_{\mathrm{g}}$, material $\sigma_{\mathrm{m}}$ and radiative $\sigma_{\mathrm{r}}\left(=\sigma_{\mathrm{g}}-\sigma_{\mathrm{m}}\right)$ parts of entropy production in comparison with Paltridge's results (extrema of $\zeta / T_{\mathrm{a}}$, where $T_{\mathrm{a}}$ is the atmospheric temperature and $\zeta$ the meridional divergence of heat flux) and observations (from Lee and Snell 1977).

short- and long-wave entropy radiation respectively. The distribution observed is similar to that found by O'Brien (1997) who uses a simple 1D model. However, as Paltridge's box model also predicts global cloud cover, there is a narrow range for the acceptable climatic states; this is indicated by vertical dotted lines in Fig. 2, which correspond to an earth without clouds (average albedo $\approx 0.16$ ) and fully covered (average albedo $\approx 0.36$ ). The global outgoing radiation obtained in earth radiation budget experiment (ERBE) measurements by satellite is also shown (Stephens and O'Brien 1993).

Results for temperature and cloud-cover in each box, found at extrema of (10) and (11), always taking the convective hypothesis into account, are shown in Figs. 3 and 4 respectively. In Fig. 3 temperatures are compared with values obtained by following Paltridge's formulation and with observations from Lee and Snell (1977). In Fig. 4 cloudcover results are compared with observations due to Beryland and Strokina quoted by Peixoto and Oort (1992). Also the result at an extremum of $\sigma_{\mathrm{r}}$ is shown. Essex (1984a) found that $\sigma_{\mathrm{r}}$ is a minimum in a grey atmosphere, basing his work on one of Planck's studies. In all the cases treated feasible climatic states are found by using the same values for the parameters as Paltridge (1978). The results for temperature, Fig. 3, are very close for all the expressions. For cloud-cover, Fig. 4, a substantial asymmetry in the solution for global entropy production is observed, because of its dependence on the radiative field which is strongly conditioned by the asymmetric distribution of surface albedo. Nevertheless, the results for $\sigma_{\mathrm{m}}$ and $\sigma_{\mathrm{r}}$ show good agreement with the observations, better than the results obtained from the expression used by Paltridge for high latitudes. However, the extremal condition for the expression proposed by Paltridge (1978) gives results closer to the observations near the equator.

Variations in the temperature field compared with Paltridge's result are, according to O'Brien and Stephens (1995), due to using the convective hypothesis as an analytical relationship between the variables and not as a computational process for finding the extremal values of latent- plus sensible-heat flux in each step. Thus, the temperatures are, 


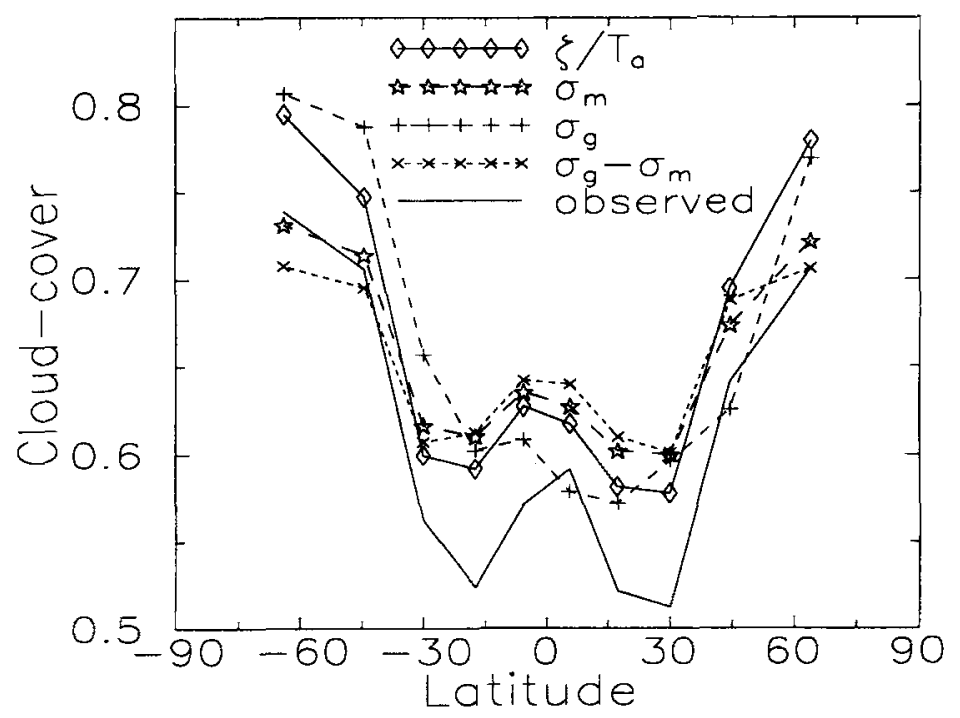

Figure 4. As Fig. 3 but for cloud cover $\theta$, obtained through extremal hypothesis. Observations in this case are from Beryland and Strokina, quoted by Peixoto and Oort (1992).

in general, $3 \mathrm{~K}$ higher according to Paltridge (1978), a problem which could be overcome by choosing suitable parameters for the long-wave emission. Differences between our study and O'Brien and Stephens (1995) arise because in our calculation the planetary albedo of cloudy and clear-sky parts strictly follows Paltridge (1978).

\section{EXTREME DYNAMICAL CASES}

Although in the present paper there is no advance in the justification of the maximumdissipation hypothesis, the results obtained for global entropy flux and material entropy production in two dynamically extreme cases may be useful for observing the predictive relevance of each expression. Thus, two completely different and unrealistic situations have been analysed; the globally averaged results are summarized in Table 1 and depicted in Figs. 5 and 6.

\section{(a) Earth without meridional heat transport}

For an earth without meridional heat transport, the system is in radiative equilibrium at any latitudinal point $(\zeta=0)$. Then, the extremal process has been carried out in reference to $\theta$ under the constraint of a whole energy balance equal to the current value (average $\theta \approx 0.62$ ). In this case, globally averaged entropy flux of radiation and material entropy production, at an extremum of $\sigma_{\mathrm{r}}$, show values close to those previously obtained. Nevertheless, the extremal procedure applied to $\sigma_{\mathrm{m}}$ and $\sigma_{\mathrm{g}}$ does not lead to any possible state coherent with the condition of global cloud cover $\approx 0.62$. $\theta=0.84$ is the minimum cloud cover in which an extremum of $\sigma_{\mathrm{m}}$ is possible, whilst the maximum using $\sigma_{\mathrm{g}}$ becomes 0.11 . Moreover, although the value obtained by $\sigma_{\mathrm{m}}$ is highly unrealistic, the globally averaged entropy flux of radiation, as well as material entropy production, do not differ greatly from those obtained in section 2. 


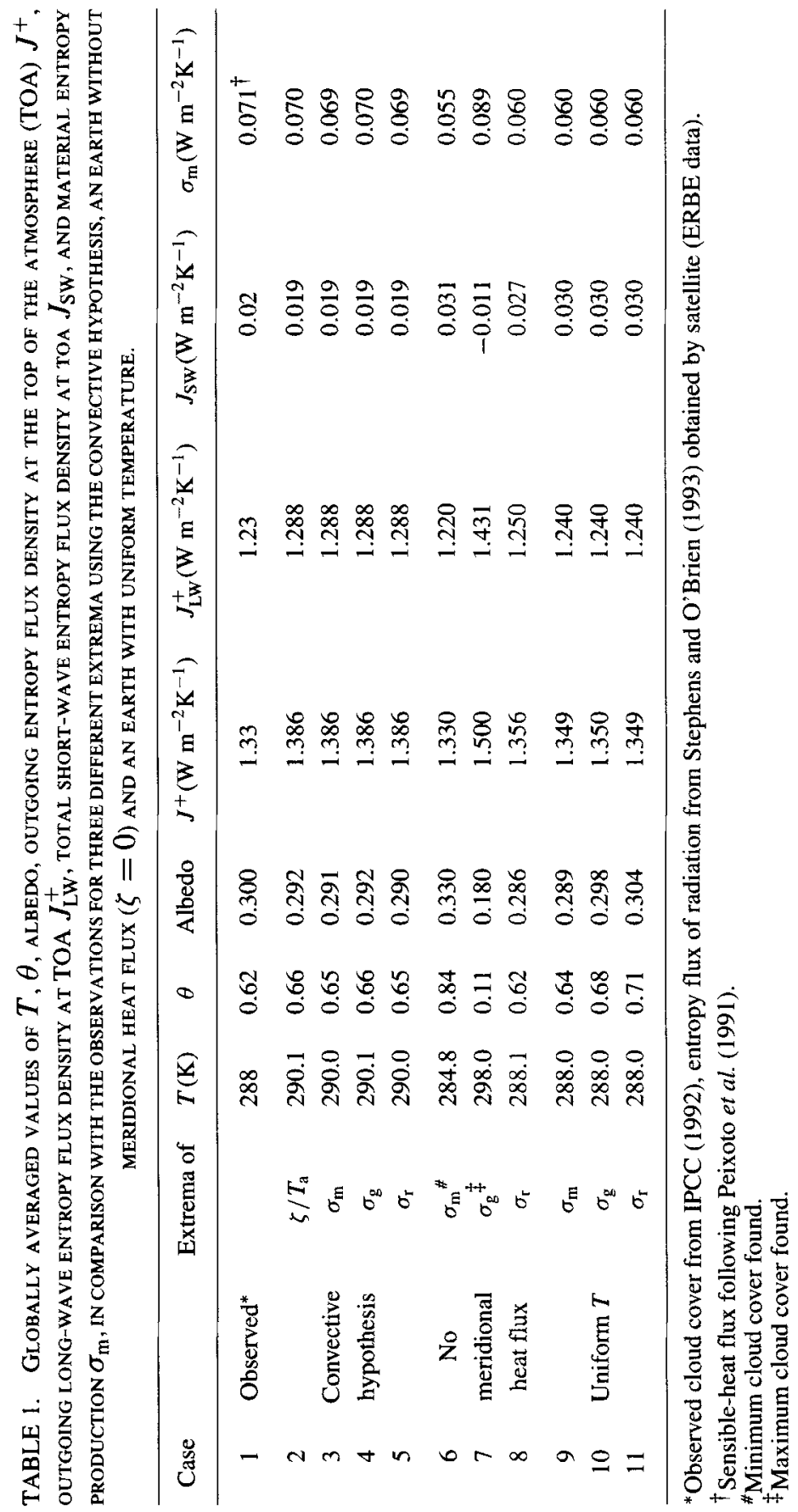




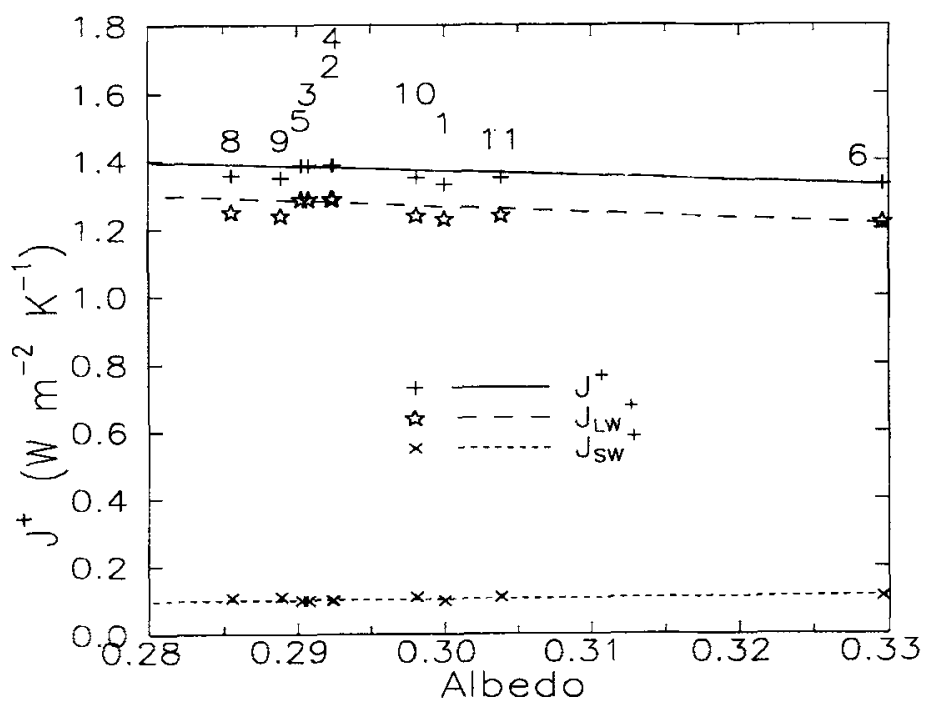

Figure 5. Globally averaged values of total outgoing entropy flux density $J^{+}$, and its long-wave $J_{\mathrm{LW}}^{+}$, and shortwave $J_{\mathrm{SW}}^{+}$, contributions for the 11 cases analysed (case 7 not included), summarized in Table 1, compared with the results obtained by using a globally averaged model (see Fig. 2).

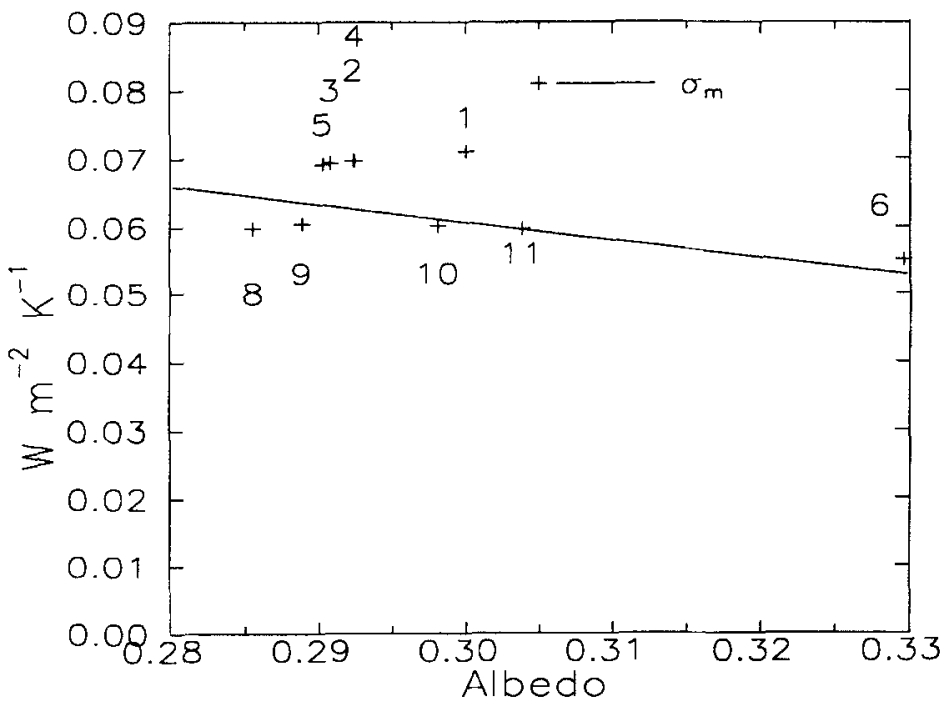

Figure 6. Globally averaged values of the material entropy production $\sigma_{m}$ for the 11 cases analysed (see Table 1; case 7 not included) compared with the results obtained using a globally averaged model (solid line).

\section{(b) Earth with uniform temperature}

For an earth with a uniform temperature $(=288 \mathrm{~K})$, the extremum of $\sigma_{\mathrm{g}}$ does not imply substantial changes in the globally averaged entropy flux of radiation. On the other hand, a climate state with averaged $\theta \approx 0.64$ is found using extrema of $\sigma_{\mathrm{m}}$, being the closest global value of albedo, for both extreme cases, to those analysed in Figs. 3 and 4. 


\section{CONCLUDING REMARKS}

Even though the contribution of the radiative field has been widely questioned with reference to its influence on the dynamics of the system, a plausible climatic state is obtained at an extremum of the entropy flux of radiation in Paltridge's box model. The material entropy production has been split into three components: meridional oceanic, atmospheric and latent- plus sensible-heat flux dissipation. Due to the box model used, the possible range of albedos has been reduced to a narrow band ( 0.16 to 0.36 ), which indicates the maximum possible variation of cloud cover. Thus, slight variations in the entropy flux of radiation, as well as in the material entropy production, are expected when changes in the dynamical description of the system are introduced. Two totally different dynamical extreme cases have been used in order to observe the variations produced in global entropy flux, in comparison with those obtained for global material entropy production. Slight deviations are found for both expressions with regard to the values obtained using the model for the present conditions. A brief summary of the results can be seen in Table 1 .

However, an averaged analysis of the results is hardly representative of the range of cases studied. Thus, all the results obtained for the two extreme cases are unrealistic if the latitudinal distribution is taken into account. This fact indicates that a globally averaged description is not enough to determine the validity of a certain expression as a predicting function within the framework of the maximum-dissipation hypothesis.

Furthermore, the results suggest that the convective hypothesis, which is not applied in the extreme cases, plays an important role in obtaining a dynamical state similar to the present one. Recently, and related to this parameter, Ozawa and Ohmura (1997) have applied an extremal condition to latent- plus sensible-entropy production, finding a reasonable vertical profile of temperatures in a $1 \mathrm{D}$ model. Nevertheless, it is obvious that the maximum-dissipation hypothesis is fundamental, since Paltridge in his earlier paper (Paltridge 1975) did not take the convective hypothesis into account and yet he obtained reasonable values for the variables (e.g. Grassl 1981).

Finally, taking advantage of the analytical relationships between the variables obtained by O'Brien and Stephens (1995) in their careful analysis of Paltridge's box model, the type of the extremum can easily be found through the calculation of the second derivatives of the expressions. With the data obtained, a maximum for global entropy production, and for its material entropy constituent is deduced, whereas a minimum is found for $\sigma_{\mathrm{r}}$ which agrees with Planck (1913) and Essex (1984a). The plausible states corresponding to $\sigma_{\mathrm{m}}$ and $\sigma_{\mathrm{r}}$ seem to be very close, implying a more abrupt peak on the extremum of $\sigma_{\mathrm{m}}$ than in $\sigma_{\mathrm{g}}$.

However, the fundamental question of why the climate system is governed by an extremal principle, if it is, remains open, in the absence of a careful theoretical analysis of a general system including material transport and radiation fields outside equilibrium.

\section{ACKNOWLEDGEMENT}

This work has been partially supported by the Ministerio de Educación y Cultura of the Spanish Government under contracts CL195-1867 and PB96-0451.

APPENDIX

Procedure to find extrema

The following development is based on the procedure used by O'Brien and Stephens 
(1995). Here, in order to find extrema of a function $\sigma\left(\zeta / T_{\mathrm{a}}, \sigma_{\mathrm{g}}, \sigma_{\mathrm{r}}\right.$ or $\left.\sigma_{\mathrm{m}}\right)$ subject to a certain global constraint $\Gamma$, a Lagrange multiplier $\beta$ is introduced, giving:

$$
\Phi_{i}=\sigma_{i}-\beta \Gamma,
$$

for the $i$ th box. By using the analytical relationships between the variables $T, \theta$ and $\zeta$, the functional $\Phi_{i}$ is expressed as a function of $\zeta$ when the convective hypothesis, or the earth with uniform temperature, is assumed. In both cases the constraint $\Gamma$ becomes:

$$
\Gamma=\sum_{i=1}^{10} \zeta_{i}=0
$$

i.e. zero storage heat flux over the whole system (composed of ten latitudinal boxes).

For the radiative equilibrium model, $\Phi_{i}$ is expressed as a function of the cloud-cover where the constraint $\Gamma$ is:

$$
\Gamma=\sum_{i=1}^{10}(A-B \theta)=\text { constant }
$$

i.e. energy balance is equal to the current value, assuming a globally averaged cloud-cover $\approx 0.62$.

The extreme of (A.1) satisfies:

$$
\frac{\mathrm{d} \Phi_{i}}{\mathrm{~d} \gamma}=0=\frac{\mathrm{d} \sigma_{i}}{\mathrm{~d} \gamma}-\beta \frac{\mathrm{d} \Gamma}{\mathrm{d} \gamma},
$$

where $\gamma=\zeta$ assuming the convective hypothesis or the uniform-temperature model, and $\gamma=\theta$ assuming the radiative equilibrium model.

Then, an arbitrary value of $\beta$ is chosen and roots of (A.4) are found. From the results, it is observed that either the constraint $\Gamma$ is satisfied, or if not $\beta$ is tuned until the values obtained through (A.4) agree with $\Gamma$.

Callies, U. and Herbert, F.

Ciancio, V. and Verhás, J.

De Groot, S. R. and Mazur, P. Essex, C.

Goody, R. and Abdou, W.

Grassl, H.

IPCC (Intergovernmental Panel on Climate Change)

Landsberg, P. T. and Tonge, G.

\section{REFERENCES}

1984 On the treatment of radiation in the entropy budget of the earthatmosphere system. Dev. Atmos. Sci., 16, 311-329

1988 Radiative processes and non-equilibrium thermodynamics. $J$. Appl. Math. Phys., 39, 242-266

1990 A thermodynamic theory for radiating heat transfer. J. NonEquilibrium Thermodyn., 15, 33-43

1991 A thermodynamic theory for heat radiation through the atmosphere. J. Non-Equilibrium Thermodyn., 16, 57-65

1984 Non-equilibrium thermodynamics. Dover, New York, USA

1984a Minimum entropy production in steady state and radiative transfer. Astrophys. J., 285, $279-293$

1984b Radiation and irreversible thermodynamics of climate. J. Atmos. Sci., 41, 1985-1991

1996 Reversible and irreversible sources of radiation entropy. $Q . J . R$. Meteorol. Soc., 122, 483-494

1981 The climate at maximum entropy production by meridional atmospheric and oceanic heat fluxes. Q.J.R. Meteorol. Soc., 107, 153-166

1992 Climate change: The supplementary report to the IPCC Scientific Assessment. Eds. J. T. Houghton, B. A. Callander and S. K. Varney, Cambridge University Press, New York, USA
Thermodynamics of the conversion of diluted radiation. J. Phys. $A, 12,551-562$ 
Lee, P. S. and Snell, F. M.

1977

Lesins, G. B.

1990

Li, J. and Chýlek, P.

1994

Li, J., Chýlek, P. and Lesins, G. B. 1994

Mobbs, S. D.

1982

Nicolis, G. and Nicolis, C.

O'Brien, D. M.

O'Brien, D. M. and Stephens, G. L. 1995

Ore, A.

Ozawa, H. and Ohmura, A.

Paltridge, G. W.

1980

1997

1955

1997

1975

1978

1979

1981

Peixoto, J. P. and Oort, A. H.

Peixoto, J. P., Oort, A. H., de Almeida, M. and Tomé, A.

Planck, $\mathrm{M}$.

Prigogine, I.

Shutts, G. H.

Stephens, G. L. and O'Brien, D. M.

Wyant, P. H., Mongroo, A. and Hameed, S.

1981

1993
An annual zonally averaged global climatic model with diffuse cloudiness feedback. J. Atmos. Sci., 34, 847-853

On the relationship between radiative entropy and temperature distributions. J. Atmos. Sci., 47, 795-803

Entropy in climate models. Part II: Horizontal structure of atmospheric entropy production. J. Atmos. Sci., 51, 1702-1708

Entropy in climate models. Part I: Vertical structure of atmospheric entropy production. J. Atmos. Sci., 51, 1691-1701

Extremal principles for global climate models. Q. J. R. Meteorol. Soc., 108, 535-550

On the entropy balance of the earth-atmosphere system. $Q . J . R$. Meteorol. Soc., 106, 691-706

A yardstick for global entropy-flux. Q. J. R. Meteorol. Soc., 123, 243-260

Entropy and climate. II: Simple models. Q.J. R. Meteorol. Soc., 121, 1773-1796

Entropy of radiation. Phys. Rev., 98, 887-888

Thermodynamics of a global-mean state of the atmosphere-a state of maximum entropy increase. J. Climate, 10, 441-445

Global dynamics and climate-a system of minimum entropy exchange. O. J. R. Meteorol. Soc., 101, 475-484

The steady-state format of global climate. Q.J.R. Meteorol. Soc., 104, $927-945$

Climate and thermodynamic systems of maximum dissipation. $\mathrm{Na}$ ture, 279, 630-631

Thermodynamic dissipation and the global climate system. $Q . J$. R. Meteorol. Soc., 107, 531-547

1992 Physics of climate. American Institute of Physics, New York, USA

1991 Entropy budget of the atmosphere. J. Geophys. Res., 96, 1098110988

1913 Heat radiation. Transl. 2nd ed. republished 1959. Dover, New York, USA

1947 Etude thermodynamique des phénomènes irreversibles. Desoer, Liège, Belgium

Maximum entropy production states in quasi-geostrophic dynamical models. Q. J. R. Meteorol. Soc., 107, 503-520

Entropy and climate. I: ERBE observations of the entropy production of the earth. Q.J.R. Meteorol. Soc., 119, 121-152

1988 Determination of the heat-transport coefficient in energy-balance climate models by extremisation of entropy production. $J$. Atmos. Sci., 45, 189-193 